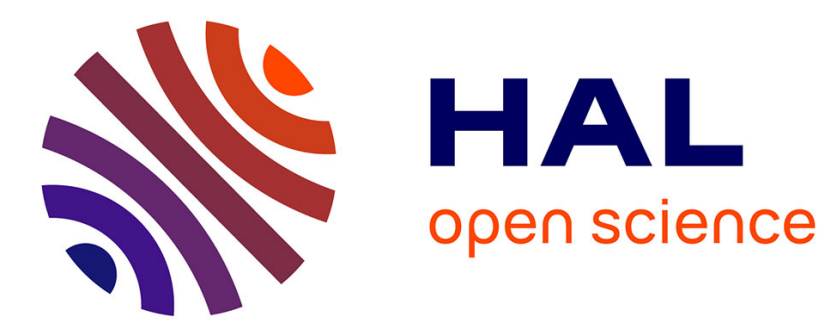

\title{
Non-Gaussian nature and entanglement of spontaneous parametric nondegenerate triple-photon generation
}

Da Zhang, Yin Cai, Zhan Zheng, David Barral, Yanpeng Zhang, Min Xiao, Kamel Bencheikh

\section{- To cite this version:}

Da Zhang, Yin Cai, Zhan Zheng, David Barral, Yanpeng Zhang, et al.. Non-Gaussian nature and entanglement of spontaneous parametric nondegenerate triple-photon generation. Physical Review A, 2021, 103 (1), 10.1103/PhysRevA.103.013704 . hal-03162654

\section{HAL Id: hal-03162654 \\ https://hal.science/hal-03162654}

Submitted on 26 Nov 2021

HAL is a multi-disciplinary open access archive for the deposit and dissemination of scientific research documents, whether they are published or not. The documents may come from teaching and research institutions in France or abroad, or from public or private research centers.
L'archive ouverte pluridisciplinaire HAL, est destinée au dépôt et à la diffusion de documents scientifiques de niveau recherche, publiés ou non, émanant des établissements d'enseignement et de recherche français ou étrangers, des laboratoires publics ou privés. 


\title{
Non-Gaussian nature and entanglement of spontaneous parametric nondegenerate triple-photon generation
}

\author{
Da Zhang, ${ }^{1,2}$ Yin Cai, ${ }^{1, *}$ Zhan Zheng, ${ }^{3}$ David Barral, ${ }^{2}$ Yanpeng Zhang $\odot,{ }^{1, \dagger}$ Min Xiao,, 4 and Kamel Bencheikh ${ }^{2,+}$ \\ ${ }^{1}$ Key Laboratory for Physical Electronics and Devices of the Ministry of Education and Shaanxi Key Lab of Information Photonic Technique, \\ School of Electronic and Information Engineering, Xi'an Jiaotong University, Xi'an 710049, China \\ ${ }^{2}$ Centre de Nanosciences et de Nanotechnologies, CNRS/Université Paris-Saclay, 91120 Palaiseau, France \\ ${ }^{3}$ Zhejiang Provincial Key Laboratory of Quantum Precision Measurement, College of Science, Zhejiang University of Technology, \\ Hangzhou 310023, China \\ ${ }^{4}$ Department of Physics, University of Arkansas, Fayetteville, Arkansas 72701, USA \\ ${ }^{5}$ National Laboratory of Solid State Microstructures and School of Physics, Nanjing University, Nanjing 210093, China
}

(Received 25 April 2020; accepted 11 December 2020; published 6 January 2021)

\begin{abstract}
We theoretically demonstrate through numerical methods that the triple-photon state generated by threephoton spontaneous parametric down-conversion is a non-Gaussian Greenberger-Horne-Zeilinger state, showing super-Gaussian statistics. Interestingly, the degree of entanglement between the modes of the triple-photon state is stronger than that corresponding to the two-mode squeezed vacuum state produced by a quadratic Hamiltonian with the same parameters. Furthermore, we propose a model to prepare two-mode sub-Gaussian entangled states with a tunable negative Wigner function based on quadrature projection measurements. We find that these Gaussian projection measurements with outcomes $X \geqslant 1$ not only improve the entanglement of the residual two modes but also introduce a Gaussian component, resulting in the coexistence of Gaussian and non-Gaussian entanglement.
\end{abstract}

DOI: 10.1103/PhysRevA.103.013704

\section{INTRODUCTION}

Early development of continuous-variable quantum information technologies (CV-QITs) mainly focused on Gaussian states and Gaussian operations [1,2], achieving remarkable accomplishments [3-5]. In CV-QITs, information is encoded in the amplitude and phase quadratures of optical fields and retrieved by homodyne detection. Nonclassical sources in CV-QITs are generally single-mode squeezed and twomode entangled states. They are Gaussian states because the associated quadratures exhibit normal distribution. The combination of Gaussian states and Gaussian operations provides a complete framework for many CV-QIT protocols [6-12]. However, there has been growing awareness of some important inherent limitations of this Gaussian framework, such as quantum distillation [13], which is an essential protocol for long-distance quantum communication, especially quantum key distribution. A prominent no-go theorem states that Gaussian operations cannot distill Gaussian states [14]. It has been demonstrated that sub-Gaussian (SG) sources are necessary to achieve universal CV quantum computation [15-19].

Since the available resources for CV-QITs are, by nature, mostly Gaussian, one must rely on degaussification via quantum nonlinear processes to produce SG resources. Ourjoumtsev et al. used photon subtraction as a non-Gaussian

\footnotetext{
*caiyin@xjtu.edu.cn

†ypzhang@mail.xjtu.edu.cn

*kamel.bencheikh@c2n.upsaclay.fr
}

operation to prepare and increase entanglement between two Gaussian states [20,21]. So far, photon subtraction and addition have been employed to prepare various optical SG states, such as superposition of coherent states [22-25], hybrid entanglement [26,27], and multimode SG states [28]. However, one of the main advantages of CV-QITs is determinism, but the probabilistic degaussification protocol losses this key feature.

A promising quantum technology that can deterministically generate SG states is the triple-photon generation (TPG) process. Douady and Boulanger [29] first demonstrated the existence of phase-matched TPG in the $\mathrm{KTiOPO}_{4}$ crystal in the regime of double seeding. Recently, the outstanding work of observing spontaneous TPG in a superconducting parametric cavity was reported by Sandbo Chang et al. [30]. Optical fiber is also a potential candidate for preparing a spontaneous triple-photon state (TPS) [31]. In a fully degenerate configuration, the Wigner function of the TPS exhibits negativities $[30,32,33]$, a clear signature of SG statistics, which is impossible to achieve by second-order down-conversion (SODC) and linear optics. Nonlinear steering has been proposed and verified based on a partially degenerate configuration [34]. González et al. revealed the relationship between Gaussian entanglement and both number of seeded modes and intensity in the nondegenerate case [35]. Very recently, work [36] has shown that the nondegenerate TPS in a spontaneous parametric regime possesses fully inseparable and genuine tripartite entanglement. However, deeper insight into the physics of nondegenerate TPS is still missing.

In this paper, we theoretically demonstrate through numerical methods that TPS is a non-Gaussian 

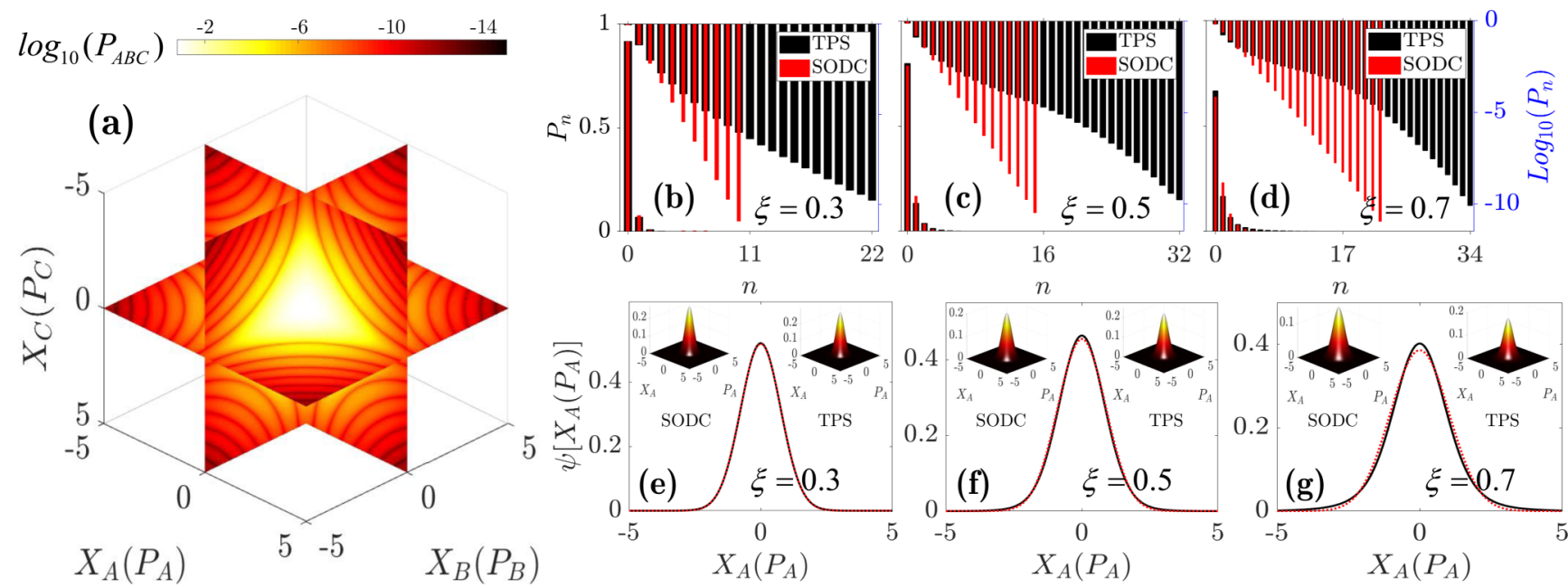

FIG. 1. (a) Quadrature joint probability distribution of modes $A, B$, and $C$ for interaction strength $\xi=\kappa t \alpha_{p}=0.3$. The photon number distribution of mode $A$ in linear and $\log$ scales for (b) $\xi=0.3$, (c) $\xi=0.5$, and (d) $\xi=0.7$, where modes $B, C$, and $P$ are traced out. Black and red histograms represent TPS and SODC. (e), (f), and (g) are, respectively, the marginal distribution of the photon number states represented by (b), (c), and (d), where the full line and the dotted line represent TPS and SODC respectively. The insets on the left and right in (e), (f), and (g) are, respectively, the Wigner functions of mode $A$ of TPS and SODC.

Greenberger-Horne-Zeilinger (GHZ) state, presenting super-Gaussian statistics. The degree of entanglement of TPS is higher than the two-mode squeezed vacuum state produced by the SODC with the same interaction parameters. We also reveal how a two-mode $\mathrm{SG}$ entangled state with tunable non-Gaussianity is prepared by performing simple quadrature projection measurements and how the entanglement and SG level of the generated states depend on the measurement of quadratures.

\section{NON-GAUSSIAN NATURE AND ENTANGLEMENT OF TPS}

We start our analysis by considering the interaction Hamiltonian describing the nondegenerate TPG process,

$$
\hat{H}=i \hbar \kappa^{(3)}\left(\hat{a}^{\dagger} \hat{b}^{\dagger} \hat{c}^{\dagger} \hat{p}-\hat{a} \hat{b} \hat{c} \hat{p}^{\dagger}\right)
$$

where $\kappa^{(3)}$ is the third-order coupling constant that describes the strength of the nonlinear interaction. The annihilation operators $\hat{a}, \hat{b}, \hat{c}$, and $\hat{p}$ describe, respectively, the three downconverted modes and the pump mode. Using this Hamiltonian, the Schrödinger equation is solved to deduce the final state of system at time $t$ when considering that the initial state is vacuum for the triplets and a coherent mode $\alpha_{p}$ for the pump. The evolution equation can be numerically solved by the Monte Carlo method [37-40]. Figure 1(a) shows the quadrature joint probability distribution corresponding to modes $A, B$, and $C$. The distribution, which was initially spherical in the 3D plotmodes $A, B$, and $C$ are initially vacuum states-is now very complex, exhibiting star shapes and interferences, indicating quantum correlations between them.

We will now discuss the quantum properties of a single mode of TPS. For comparison, we also present the photon number distribution of the twin modes generated by SODC and described by the Hamiltonian $H_{S}=i \hbar \kappa^{(2)}\left(\hat{a}^{\dagger} \hat{b}^{\dagger} \hat{p}-\right.$ $\left.\hat{a} \hat{b} \hat{p}^{\dagger}\right)$, where $\kappa^{(2)}$ is the second-order nonlinear interaction strength. To facilitate the comparison of the physical properties of TPS and SODC, we assume $\kappa^{(2)}=\kappa^{(3)}=\kappa$. Figures 1(b), 1(c), and 1(d) show the photon number distribution of mode $A$ in linear and $\log$ scales for interaction strengths $\xi=0.3,0.5$, and 0.7 , where $\xi=\kappa t \alpha_{p}$. These processes could be obtained by considering the third-order nonlinear material in a high-finesse cavity or an optimized superconducting parametric cavity. These photon number distributions of TPS (in black) are quite different from the exponential decay of SODC (in red), the Poisson distribution of coherent states, or the Bose-Einstein distribution of thermal states [41,42]. With the increasing of the interaction time, the shape of the photon number distribution of TPS gradually goes from an arc to an " $\backsim$ " shape in the log scale, whereas it is always linear for SODC. This nonlinear photon number distribution has implications on the marginal distributions (full line) represented in Figs. 1(e), 1(f), and 1(g) along with the Gaussian marginal distribution (dotted line) of SODC. As the interaction strength increases, the marginal distribution of TPS becomes superGaussian, exhibiting a higher peak, narrower shoulders, and longer tails. The TPS displays a super-Gaussian statistics in the quadratures, demonstrating thus the non-Gaussian nature of nondegenerate TPS.

After studying the single-mode properties of the TPS, we focus on its multimode SG and entanglement features. As shown in Figs. 1(e), 1(f), and 1(g), it is difficult to qualitatively grasp the super-Gaussian nature of the TPS from the Wigner function. This is in contrast to photon subtraction or addition, where the negativity of the Wigner function at the origin of the phase space is a signature of SG. Thus, a measure of non-Gaussianity based on a different model is necessary here. We employ the quantum relative entropy (QRE) to quantify the super-Gaussian nature of the TPS. The QRE between a given quantum state $\varrho$ and a reference Gaussian state $\gamma$ is defined as follows [43]:

$$
\delta[\varrho]=E_{v N}(\gamma)-E_{v N}(\varrho),
$$



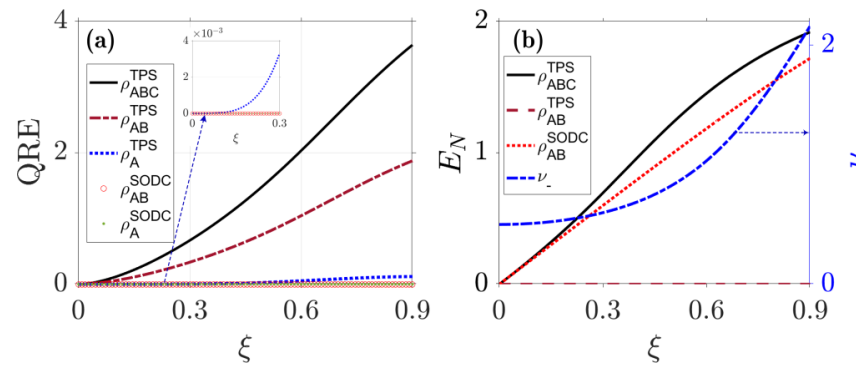

FIG. 2. (a) QRE and (b) entanglement of states generated by TPG and SODC, respectively. $\nu_{-}$is the smallest symplectic eigenvalue of the partially transposed covariance matrix of the TPS.

where $E_{v N}(\varrho)=\operatorname{Tr}[\varrho \ln \varrho]$ is the von Neumann entropy (VNE) and $\gamma$ has the same covariance matrix and the first moments as $\varrho$. The VNE of a Gaussian state with covariance matrix $\gamma$ and symplectic eigenvalue $v_{j}$ is

$$
E_{v N}(\gamma)=\sum_{j=1}^{n} f\left(v_{j}\right),
$$

where $f(x)=\left(x+\frac{1}{2}\right) \ln \left(x+\frac{1}{2}\right)-\left(x-\frac{1}{2}\right) \ln \left(x-\frac{1}{2}\right)$.

Let us first consider the SODC process. The VNE (entanglement entropy) of the two-mode squeezed vacuum is [41]

$$
E_{v N}^{S}=\cosh ^{2}(\xi) \ln \cosh ^{2}(\xi)-\sinh ^{2}(\xi) \ln \sinh ^{2}(\xi) .
$$

Figure 2(a) shows in circles the QRE $\left[\delta\left(\varrho_{A B}^{\mathrm{SODC}}\right)=\right.$ $\left.E_{v N}^{S}\left(\gamma_{A B}\right)-E_{v N}^{S}\right]$ of SODC (see the Appendix for details), where $E_{v N}^{S}\left(\gamma_{A B}\right)$ represents the VNE of a reference two-mode Gaussian state. Not surprisingly, it is equal to zero for all interaction times since $\gamma_{A B}=\varrho_{A B}^{\mathrm{SODC}}$. Any single-mode element of SODC is in a thermal state, and the VNE is $E_{v N}^{S}\left(\varrho_{A / B}\right)=E_{v N}^{S}$. Similarly, the QRE of a single mode $\left[\delta\left(\varrho_{A}^{\text {SODC }}\right)\right]$ is equal to zero, as shown in Fig. 2(a) by the dots. Thus, for every Gaussian state, independent of the number of modes, the QRE is zero.

When the interaction strength is small, the TPS can be approximated as $\left|\psi_{T}\right\rangle \approx\left(|000\rangle+\xi|111\rangle+\xi^{2} / 2|222\rangle\right)_{A B C}$. The entanglement entropy of the TPS is still equal to the VNE of each subsystem. Due to the symmetry of the system, we obtain $E_{v N}^{T}\left(\rho_{A / B / C}\right)=E_{v N}^{T}\left(\rho_{A B / B C / A C}\right)$. Thus, the QRE mainly depends on the VNE of the reference Gaussian state. For the TPS represented by $\left|\psi_{T}\right\rangle$, the covariance matrix related to the reference Gaussian state is $\gamma_{A B C}=\operatorname{Diag}(\lambda, \cdots, \lambda)$, where $\lambda=3 \xi^{2}+5 \xi^{4} / 4$. The VNE of the reference Gaussian state satisfies the relationship $E_{v N}^{T}\left(\gamma_{A B C}\right)=3 E_{v N}^{T}\left(\gamma_{A B / B C / A C}\right) / 2=$ $3 E_{v N}^{T}\left(\gamma_{A / B / C}\right)$. Therefore, we can deduce a mathematical relation for the QRE between the TPS and its subsystems, given by

$$
\delta^{T}\left(\rho_{A B C}\right)-\delta^{T}\left(\rho_{A B}\right)=\delta^{T}\left(\rho_{A B}\right)-\delta^{T}\left(\rho_{A}\right) .
$$

Figure 2(a) displays the evolution of QRE as a function of the interaction strength. We see that the QRE of TPS increases monotonically throughout the interaction time. For its subsystems $\rho_{A B}$ and $\rho_{A}$, their QRE still exists, as shown in Fig. 2(a) by the dotted-dashed and dotted lines, respectively. The different results in Fig. 2(a) are calculated numerically without any assumption about the evolved state, validating the relation of Eq. (5).
Next, we show that the TPS is not only super-Gaussian but also entangled. As an entanglement witness we use the logarithmic negativity defined as $E_{N}=\ln \left\|\varrho^{T_{A}}\right\|_{1}$ [44], where $\varrho^{T_{A}}$ is the partial transpose over mode $A$ and $\|\cdot\|_{1}$ is the trace norm. Obviously, modes $A, B$, and $C$ are entangled. This is shown in the logarithmic negativity plots in Fig. 2(b) (full line). In addition, the minimum symplectic eigenvalue $v_{-}$of the partially transposed covariance matrix of TPS is greater than or equal to $1 / 2\left[\hat{a}=\left(\hat{X}_{A}+i \hat{P}_{A}\right) / \sqrt{2}\right]$ for all interaction times [dashed line in Fig. 2(b)], which means that there is no Gaussian entanglement among the modes $A, B$, and $C$ [45]. This is consistent with the experimental results [30]. The reason for this is that the quantum correlations of the TPS are purely super-Gaussian, not detected by Gaussian-type inseparability criteria. Higher-order cumulants need to be calculated for non-Gaussian statistics. Remarkably, for the same interaction strength, although the TPS contains more vacuum noise, its degree of entanglement is higher than that of the SODC. Another feature is that when one mode is traced out, the remaining two modes are not entangled, although they are super-Gaussian, which is also a distinctive signature of the GHZ state. The evolution of the entanglement of the TPS versus the losses is discussed in the Appendix.

Experimentally, the numerical results can be studied in a resonant configuration where the third-order nonlinear interaction is enhanced using a cavity. To fully characterize TPS, we can use the optical tomographic method [46-48]. Assuming that modes $A, B$, and $C$ are selected close to the degeneracy point $\left(\omega_{p} / 3\right)$, a single classical field oscillating at $\omega_{p} / 3$ can be used as a local oscillator for the three homodyne detections. Such a field could be easily locked in phase to the pump of the TPG after producing a field at $\omega_{p}$ through third-harmonic generation. Our theoretically analysis could also be implemented in the radio-frequency regime following the recent results in Ref. [30].

\section{TWO-MODE ENTANGLED STATE WITH A TUNABLE NEGATIVE WIGNER FUNCTION}

Entangled states with negative Wigner functions have been proven to be the key to speed up computation in quantum computing [49]. Nowadays, photon addition and subtraction are the most effective ways to prepare two-mode SG states exhibiting negative Wigner functions. However, to the best of our knowledge, the SG properties produced by these methods are not readily tunable. In our proposal, the projection induced by the measurement of a quadrature allows the SG properties to be tuned continuously. Experimentally, the projection measurement can be regarded as the postselection processing of the reconstructed density matrix. Figure 3(a) shows the dynamic evolution of the QRE of the conditional two-mode $\left(\varrho_{A B}^{C}\right)$ and arbitrary single-mode $\left(\varrho_{A / B}^{C}\right)$ states versus the projection measurement $X_{C}$ of mode $C$. For the outcome $X_{C}=0$ in the homodyne detection, the probability distribution is the largest, and $\delta\left[\varrho_{A B}^{C}\right]$ is greater than zero. In the vicinity of $X_{C}=0$, as $\left|X_{C}\right|$ increases, the photon number distribution of $\varrho_{A B}^{C}$ gradually tends to decay exponentially, making $\delta\left[\varrho_{A B}^{C}\right]$ decrease. This is a gaussification process. As $\left|X_{C}\right|$ continues to increase, $\varrho_{A B}^{C}$ starts to deviate from the exponential decay distribution; thus, the QRE increases significantly, corresponding 

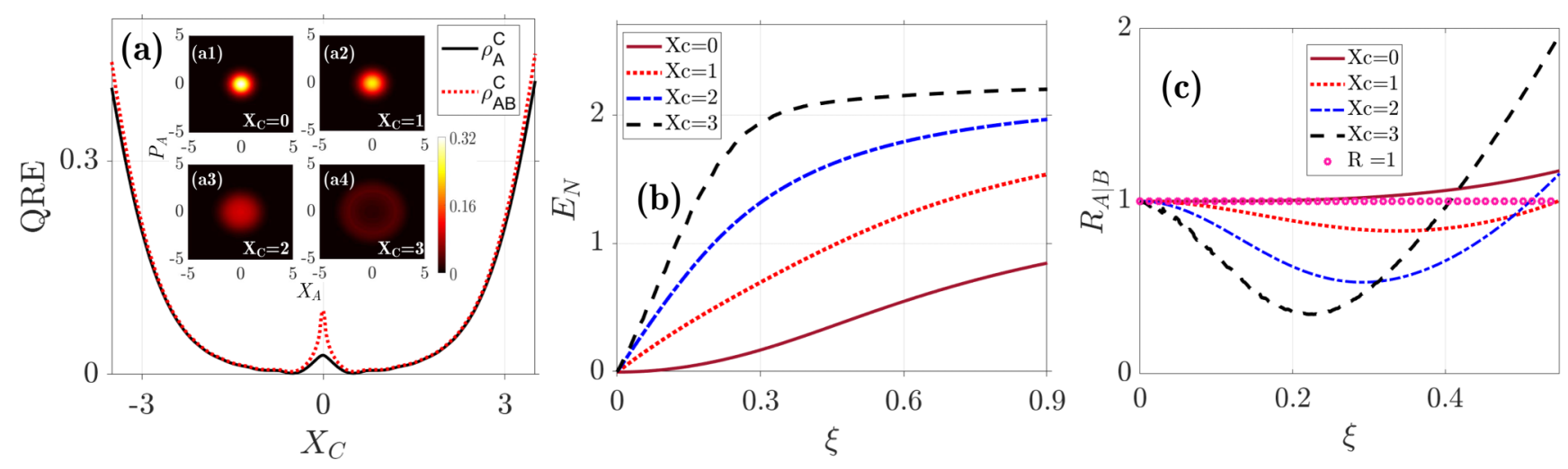

FIG. 3. (a) The QRE of conditional two-mode $\rho_{A B}^{C}$ (dotted line) and $\rho_{A}^{C}$ (full line) versus $X_{C}$ when $\xi=0.3$. The insets show the Wigner functions of projected mode $A$ when (a1) $X_{C}=0$, (a2) 1, (a3) 2, and (a4) 3 (mode $B$ is traced out). (b) The logarithmic negativity is greater than zero for all projection measurements, indicating entanglement between modes $A$ and $B$. (c) The quadratures display EPR steering when $X_{C} \geqslant 1$ and are not EPR steerable when $X_{C}=0$. The jitter in (b) and (c) is caused by a lower probability of projection measurement when $\xi$ is small.

to the degaussification process. The QRE of mode $A$ shows an evolution law similar to that of $\delta\left[\varrho_{A B}^{C}\right]$ if mode $B$ is traced out. In insets (a1), (a2), (a3), and (a4) of Fig. 3(a), we plot the Wigner functions of mode $A$ for values of $X_{C}=0,1,2$, and 3 , respectively. The SG signature exhibited by the Wigner functions becomes more and more obvious [from inset (a2) to (a4)], although no negativity appears.

In addition, $\varrho_{A B}^{C}$ is inseparable and describes entanglement between modes $A$ and $B$, as shown in Fig. 3(b), representing the logarithmic negativity $E_{N}$ as a function of the interaction strength and for different $X_{C}$ outcomes. With fixed interaction
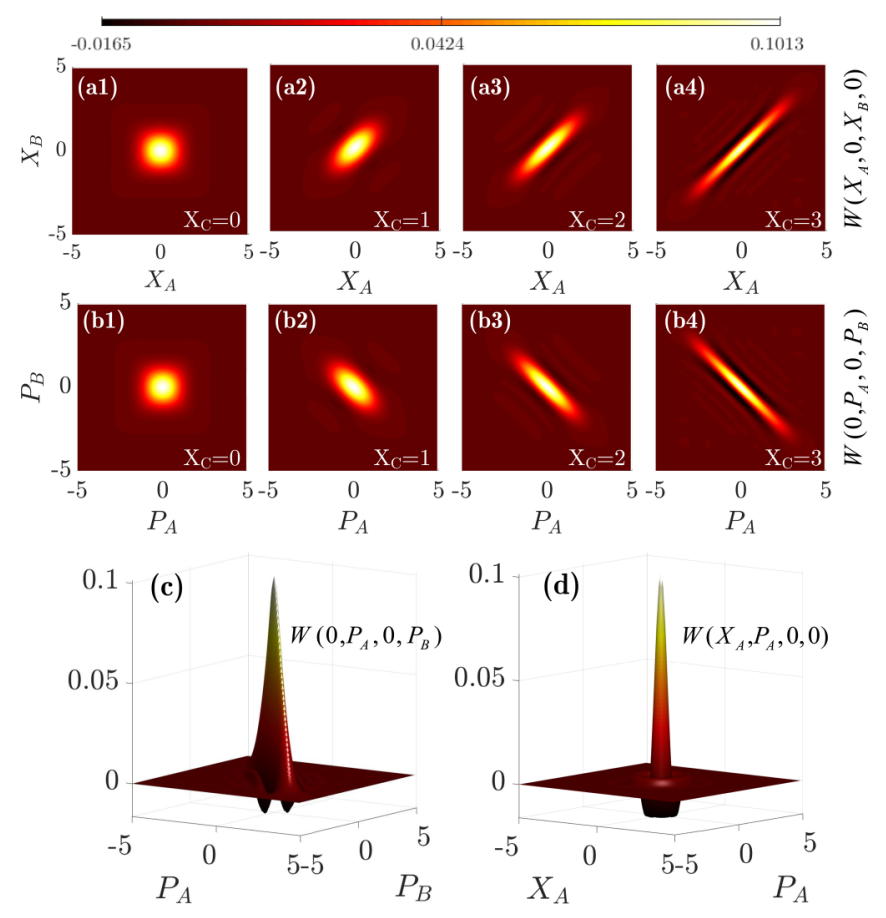

FIG. 4. Wigner functions of conditional two-mode states. (a1)-(a4) are the Wigner functions $W\left(X_{A}, 0, X_{B}, 0\right)$ for values of $X_{C}=0,1,2$, and 3, respectively. (b1)-(b4) are the same as (a1)-(a4), but for $W\left(0, P_{A}, 0, P_{B}\right)$. (c) and (d) are 3D plots of $W\left(0, P_{A}, 0, P_{B}\right)$ and $W\left(X_{A}, P_{A}, 0,0\right)$ when $X_{C}=3$, respectively. strength, $E_{N}\left(\varrho_{A B}^{C}\right)$ increases monotonically with the increase of $X_{C}$. However, compared to the TPS, $E_{N}\left(\varrho_{A B}^{C}\right)$ is reduced when $X_{C}=0$. This is because the projection measurement $X_{C}=0$ will increase the vacuum noise. For $X_{C} \geqslant 1, E_{N}\left(\varrho_{A B}^{C}\right)$ is significantly increased compared to the TPS. For comparison, we studied the linear steering of $\varrho_{A B}^{C}$ [50], defined as $R_{A \mid B}=\Delta\left(X_{A}+g_{B, X} X_{B}\right) \Delta\left(P_{A}+g_{B, P} P_{B}\right)<1$, where $g_{B, X}$ and $g_{B, P}$ are optimized real numbers. The quadrature mode $A$ is steerable by mode $B$ if $R_{A \mid B}<1$. In Fig. 3(c), we see that the quadratures of mode $A$ is steerable by $B$ within a short interaction time when the projection measurement outcomes are $X_{C}=1,2$, and 3. Interestingly, we find that $R_{A \mid B}$ is always greater than 1 under $X_{C}=0$; that is, there is only non-Gaussian entanglement between modes $A$ and $B$. Considering that the TPS contains only non-Gaussian entanglement, the homodyne detection $X_{C}=1,2$, and 3 introduce the Gaussian component to $\varrho_{A B}^{C}$, resulting in the coexistence of non-Gaussian and Gaussian entanglements.
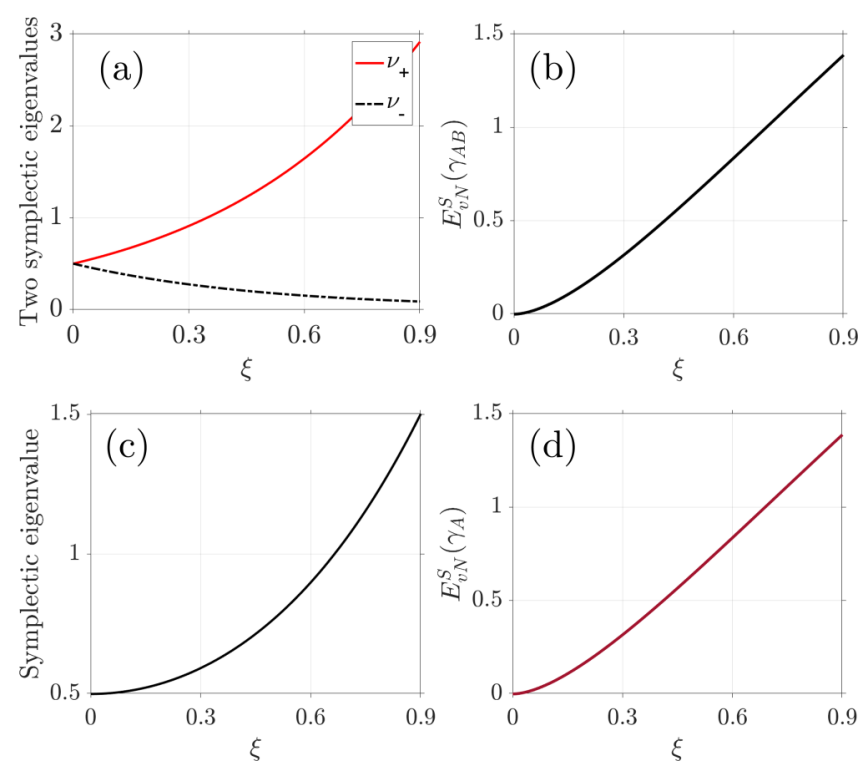

FIG. 5. (a) Evolution of two symplectic eigenvalues of $\gamma_{A B}$ and (b) its VNE. (c) and (d) are the same as (a) and (b), but for $\gamma_{A}$. 

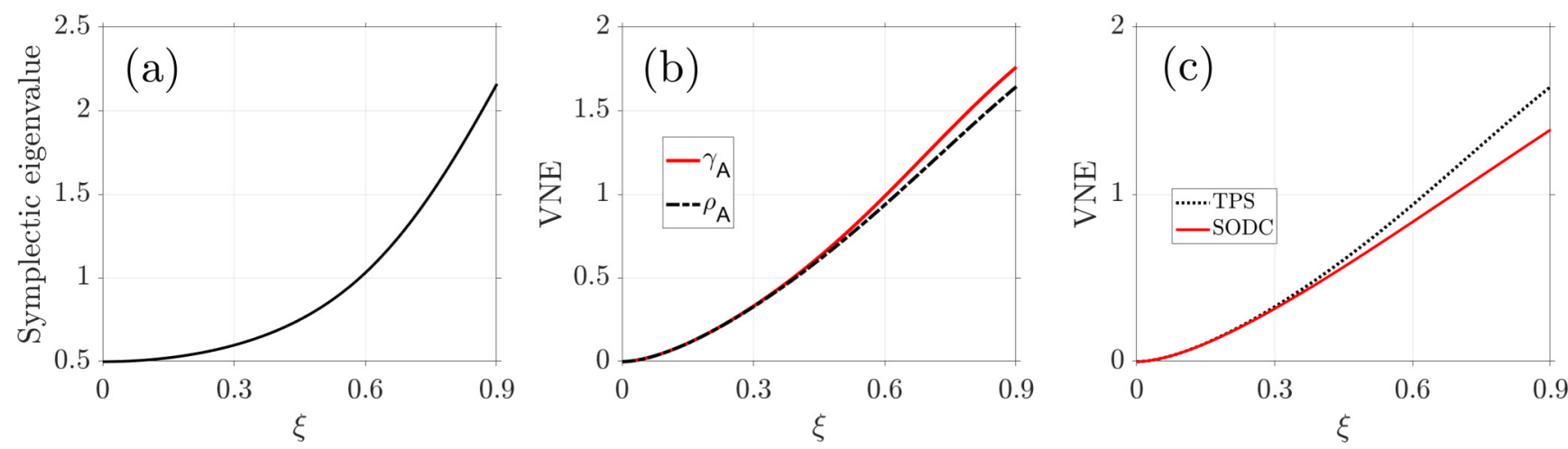

FIG. 6. (a) Evolution of the symplectic eigenvalue of $\gamma_{A B C}$. (b) The VNE of the single mode of the TPS (dotted-dashed line) and the VNE of its reference Gaussian state (full line). (c) The VNE of TPS (dotted line) and SODC (full line).

To visualize the dynamic evolution of SG and entanglement characteristics of $\varrho_{A B}^{C}$ with the projection measurement, we plot the Wigner function of $\varrho_{A B}^{C}$ for the values of $X_{C}=0,1,2$ and 3 in Fig. 4. For an outcome on $X_{C}=0$, $W\left(X_{A}, 0, X_{B}, 0\right)$ [see Fig. 4(a1)] and $W\left(0, P_{A}, 0, P_{B}\right)$ [see Fig. 4(b1)] reveal quadruple symmetry (super-Gaussian) and that the amplitude and phase quadratures do not exhibit any correlation, which is also confirmed by the steering in Fig. 3(c). For the values of $X_{C}=1,2$, and 3, $W\left(X_{A}, 0, X_{B}, 0\right)$ [from Figs. 4(a2) to 4(a4)] shows that the amplitude quadratures are correlated, while the phase quadratures are anticorrelated in $W\left(0, P_{A}, 0, P_{B}\right)$ [from Figs. 4(b2) to 4(b4)]. Importantly, the correlation gradually increases with the increase of $X_{C}$. Finally, we also show 3D plots of $W\left(0, P_{A}, 0, P_{B}\right)$ and $W\left(X_{A}, P_{A}, 0,0\right)$ for an outcome on $X_{C}=$ 3 in Figs. 4(c) and 4(d). The Wigner function shows clearly negative values which are real evidence of the SG nature of the generated states.

\section{CONCLUSION}

In summary, we have theoretically revealed that the triple-photon state produced by triple-photon generation is a pure super-Gaussian GHZ state, robust to optical losses. We

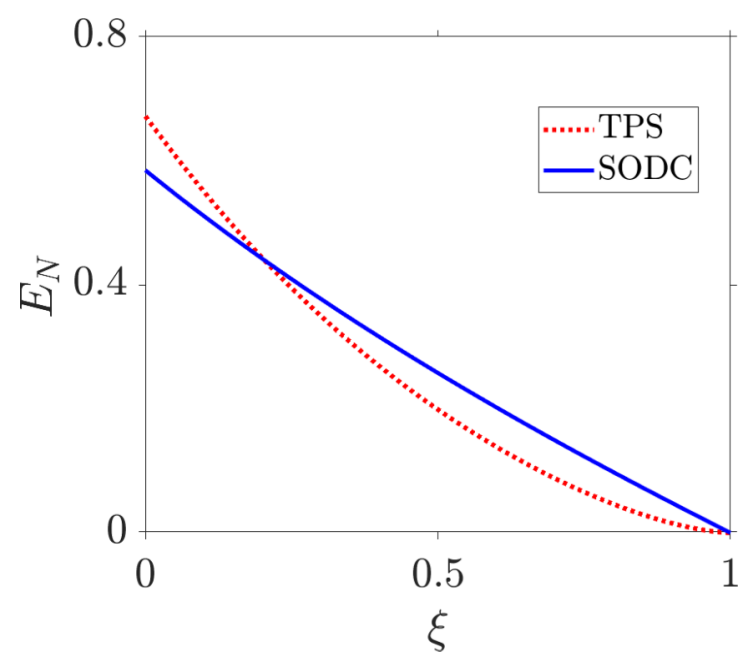

FIG. 7. Evolution of the logarithmic negativity $E_{N}$ of TPS as a function of the optical losses for $\xi=0.3$. have proposed to use projection measurements to prepare two-mode sub-Gaussian entangled states with a tunable negative Wigner function. Our results extend the boundaries of quantum optics and have potential applications in quantum information processing and quantum computing.

\section{ACKNOWLEDGMENTS}

The authors gratefully acknowledge A. Levenson for helpful discussions. This work was supported by the National Key Research and Development Program of China (Grants No. 2017YFA0303700, No. 2018YFA0307500), by the National Natural Science Foundation of China (Grants No. 61975159, No. 61605154, No. 11604256, No. 11804267, No. 11904279), and by the Agence Nationale de la Recherche through Project TRIQUI (No. ANR 17-CE24-0041).

\section{APPENDIX}

We show the intermediate results of calculating the QRE and the effects of losses on the entanglement of the TPS.

\section{Quantum relative entropy}

It is well known that Gaussian states can be fully characterized by their covariance matrix and their first-order momenta, the mean values of their quadratures. Therefore, for any quantum state $\rho$, we define the reference Gaussian state $\gamma$ with the same covariance matrix and first-order momenta as the quantum state $\rho$. For the two-mode squeezed vacuum state generated by SODC, we express its reference Gaussian state as $\gamma_{A B}$. In Fig. 5(a), we show the evolution of the two symplectic eigenvalues of $\gamma_{A B}$ as a function of the interaction strength. The corresponding VNE $E_{v}^{S}\left(\gamma_{A B}\right)$, as defined by Eq. (3) of the main text, is represented in Fig. 5(b). For a two-mode squeezed vacuum state the VNE corresponds to its entanglement entropy. Therefore, the QRE of SODC is equal to zero and is independent of the interaction strength. Similarly, we can define the reference Gaussian of any of the two modes of SODC. The density matrix of such a mode is denoted by $\rho_{A}^{\text {SODC }}$, and its reference Gaussian state is denoted by $\gamma_{A}$. Figures $5(\mathrm{c})$ and $5(\mathrm{~d})$ show, respectively, the symplectic eigenvalues of $\gamma_{A}$ and the corresponding VNE. Obviously, $\rho_{A}^{\text {SODC }}$ is a thermal state, and its QRE is equal to zero too. 
The form of the covariance matrix of the reference Gaussian state of the TPS is $\gamma_{A B C}=\operatorname{Diag}(v, \ldots, v)$, where $v$ is a variable that depends on the interaction strength. Therefore, the symplectic eigenvalue of $\gamma_{A B C}$ is $v$. In Fig. 6(a), we show the evolution of $v$ as a function of the interaction strength. The VNE of an arbitrary single mode in the TPS (dotted-dashed line) and the VNE of its reference Gaussian state (full line) are shown in Fig. 6(b). The QRE of any single mode in the TPS is not equal to zero, as shown in Fig. 2(a). On the other hand, the comparison of the VNE between the TPS and the SODC is shown in Fig. 6(c). We see that the VNE of the TPS is higher than that of the SODC, which shows that the degree of entanglement of the TPS is higher than that of SODC.

\section{Optical losses in triple-photon generation}

In quantum optics experiments, optical losses acting on the system are unavoidable and will lead to the degradation of quantum properties. In general, the calculation of loss can be modeled by placing a virtual beam splitter in front of an ideal detection system. Here, for simplicity, we assume that the losses of three down-conversion modes are the same. Considering that the initial TPS has the form $|\psi\rangle=\sum_{n=0}^{n} c_{n}|n n n\rangle$, the expression of the measured TPS is modified as

$$
\begin{aligned}
\rho_{a b c}^{\prime}= & \sum_{n} c_{n}^{\prime} \sum_{m} c_{m}^{\prime}\left[\left(\sum_{k=0}^{\min (m, n)} C_{n}^{k} C_{m}^{k} \sqrt{(n-k) !(m-k) ! k !} \sqrt{1-\eta}^{m+n-2 k} \times \eta^{k}|n-k\rangle\langle m-k|\right)_{a}\right. \\
& \left(\otimes \sum_{k=0}^{\min (m, n)} C_{n}^{k} C_{m}^{k} \sqrt{(n-k) !(m-k) !}^{\prime} ! \sqrt{1-\eta}^{m+n-2 k} \eta^{k}|n-k\rangle\langle m-k|\right)_{b} \\
& \left.\otimes\left(\sum_{k=0}^{\min (m, n)} C_{n}^{k} C_{m}^{k} \sqrt{(n-k) !(m-k) !}^{\left(n ! \sqrt{1-\eta}^{m+n-2 k}\right.} \eta^{k}|n-k\rangle\langle m-k|\right)_{c}\right],
\end{aligned}
$$

where $c_{j}^{\prime}=c_{j} / \sqrt{j !^{3}}, C_{n(m)}^{k}$ is the binomial coefficient, and $\eta$ is the reflection coefficient of the beam splitter. In Fig. 7, we show the evolution of logarithmic negativity $E_{N}$ of TPS and SODC as a function of optical losses, where $\xi=0.3$. We see that the degree of entanglement of TPS after 7\% loss per mode reaches the level of entanglement of lossless SODC. Such robustness is due to the super-Gaussian statistics of TPS. More interestingly, if the loss experienced by each mode is less than $20 \%$, the entanglement of TPS is higher than that of the SODC; otherwise, the entanglement of the TPS is lower than that of the SODC.

[1] S. L. Braunstein and P. van Loock, Rev. Mod. Phys. 77, 513 (2005).

[2] C. Weedbrook, S. Pirandola, R. García-Patrón, N. J. Cerf, T. C. Ralph, J. H. Shapiro, and S. Lloyd, Rev. Mod. Phys. 84, 621 (2012).

[3] A. Furusawa, J. L. Sørensen, S. L. Braunstein, C. A. Fuchs, H. J. Kimble, and E. S. Polzik, Science 282, 706 (1998).

[4] F. Grosshans, G. Van Assche, J. Wenger, R. Brouri, N. J. Cerf, and P. Grangier, Nature (London) 421, 238 (2003).

[5] H. Yonezawa, T. Aoki, and A. Furusawa, Nature (London) 431, 430 (2004).

[6] S. L. Braunstein and H. J. Kimble, Phys. Rev. Lett. 80, 869 (1998).

[7] T. C. Ralph and P. K. Lam, Phys. Rev. Lett. 81, 5668 (1998).

[8] S. L. Braunstein and H. J. Kimble, Phys. Rev. A 61, 042302 (2000).

[9] T. C. Ralph and E. H. Huntington, Phys. Rev. A 66, 042321 (2002).

[10] M. Ban, J. Opt. B 1, L9 (1999).

[11] N. J. Cerf, M. Lévy, and G. Van Assche, Phys. Rev. A 63, 052311 (2001).

[12] V. Scarani, H. Bechmann-Pasquinucci, N. J. Cerf, M. Dušek, N. Lütkenhaus, and M. Peev, Rev. Mod. Phys. 81, 1301 (2009).

[13] H. Takahashi, J. S. Neergaard-Nielsen, M. Takeuchi, M. Takeoka, K. Hayasaka, A. Furusawa, and M. Sasaki, Nat. Photonics 4, 178 (2010).
[14] J. Eisert, S. Scheel, and M. B. Plenio, Phys. Rev. Lett. 89, 137903 (2002).

[15] S. Lloyd and S. L. Braunstein, Phys. Rev. Lett. 82, 1784 (1999).

[16] S. D. Bartlett, B. C. Sanders, S. L. Braunstein, and K. Nemoto, Phys. Rev. Lett. 88, 097904 (2002).

[17] N. C. Menicucci, P. van Loock, M. Gu, C. Weedbrook, T. C. Ralph, and M. A. Nielsen, Phys. Rev. Lett. 97, 110501 (2006).

[18] M. Ohliger, K. Kieling, and J. Eisert, Phys. Rev. A 82, 042336 (2010).

[19] M. Ohliger and J. Eisert, Phys. Rev. A 85, 062318 (2012).

[20] A. Ourjoumtsev, A. Dantan, R. Tualle-Brouri, and P. Grangier, Phys. Rev. Lett. 98, 030502 (2007).

[21] A. Ourjoumtsev, F. Ferreyrol, R. Tualle-Brouri, and P. Grangier, Nat. Phys. 5, 189 (2009).

[22] A. Ourjoumtsev, R. Tualle-Brouri, J. Laurat, and P. Grangier, Science 312, 83 (2006).

[23] D. V. Sychev, A. E. Ulanov, A. A. Pushkina, M. W. Richards, I. A. Fedorov, and A. I. Lvovsky, Nat. Photonics 11, 379 (2017).

[24] T. Serikawa, J.-i. Yoshikawa, S. Takeda, H. Yonezawa, T. C. Ralph, E. H. Huntington, and A. Furusawa, Phys. Rev. Lett. 121, 143602 (2018).

[25] N. Biagi, L. S. Costanzo, M. Bellini, and A. Zavatta, Phys. Rev. Lett. 124, 033604 (2020).

[26] H. Jeong, A. Zavatta, M. Kang, S.-W. Lee, L. S. Costanzo, S. Grandi, T. C. Ralph, and M. Bellini, Nat. Photonics 8, 564 (2014). 
[27] O. Morin, K. Huang, J. Liu, H. Le Jeannic, C. Fabre, and J. Laurat, Nat. Photonics 8, 570 (2014).

[28] Y.-S. Ra, A. Dufour, M. Walschaers, C. Jacquard, T. Michel, C. Fabre, and N. Treps, Nat. Phys. 16, 144 (2020).

[29] J. Douady and B. Boulanger, Opt. Lett. 29, 2794 (2004).

[30] C. W. Sandbo Chang, C. Sabín, P. Forn-Díaz, F. Quijandría, A. M. Vadiraj, I. Nsanzineza, G. Johansson, and C. M. Wilson, Phys. Rev. X 10, 011011 (2020).

[31] A. Cavanna, J. Hammer, C. Okoth, E. Ortiz-Ricardo, H. CruzRamirez, K. Garay-Palmett, A. B. U'Ren, M. H. Frosz, X. Jiang, N. Y. Joly, and M. V. Chekhova, Phys. Rev. A 101, 033840 (2020).

[32] T. Felbinger, S. Schiller, and J. Mlynek, Phys. Rev. Lett. 80, 492 (1998).

[33] K. Bencheikh, F. Gravier, J. Douady, A. Levenson, and B. Boulanger, C. R. Phys. 8, 206 (2007).

[34] Y. Shen, S. M. Assad, N. B. Grosse, X. Y. Li, M. D. Reid, and P. K. Lam, Phys. Rev. Lett. 114, 100403 (2015).

[35] E. A. Rojas González, A. Borne, B. Boulanger, J. A. Levenson, and K. Bencheikh, Phys. Rev. Lett. 120, 043601 (2018).

[36] A. Agustí, C. W. Sandbo Chang, F. Quijandría, G. Johansson, C. M. Wilson, and C. Sabín, Phys. Rev. Lett. 125, 020502 (2020).

[37] J. Dalibard, Y. Castin, and K. Mølmer, Phys. Rev. Lett. 68, 580 (1992).
[38] K. Mølmer, Y. Castin, and J. Dalibard, J. Opt. Soc. Am. B 10, 524 (1993).

[39] J. Johansson, P. Nation, and F. Nori, Comput. Phys. Commun. 184, 1234 (2013).

[40] J. Johansson, P. Nation, and F. Nori, Comput. Phys. Commun. 183, 1760 (2012).

[41] P. Kok and B. W. Lovett, Introduction to Optical Quantum Information Processing (Cambridge University Press, Cambridge, 2010).

[42] G. S. Agarwal, Quantum Optics (Cambridge University Press, Cambridge, 2012).

[43] M. G. Genoni, M. G. A. Paris, and K. Banaszek, Phys. Rev. A 78, 060303(R) (2008).

[44] G. Vidal and R. F. Werner, Phys. Rev. A 65, 032314 (2002).

[45] R. Simon, Phys. Rev. Lett. 84, 2726 (2000).

[46] A. I. Lvovsky and M. G. Raymer, Rev. Mod. Phys. 81, 299 (2009).

[47] A. I. Lvovsky, J. Opt. B 6, S556 (2004).

[48] J. Řeháček, Z. Hradil, E. Knill, and A. I. Lvovsky, Phys. Rev. A 75, 042108 (2007).

[49] A. Mari and J. Eisert, Phys. Rev. Lett. 109, 230503 (2012).

[50] S. Armstrong, M. Wang, R. Y. Teh, Q. Gong, Q. He, J. Janousek, H.-A. Bachor, M. D. Reid, and P. K. Lam, Nat. Phys. 11, 167 (2015). 